\title{
The impact of 21-hydroxylase deficiency on cardiac repolarization changes in children with 21 -hydroxylase- deficient congenital adrenal hyperplasia
}

\author{
Hüseyin Anıl Korkmaz¹, Rahmi Özdemir², Mehmet Küçük², Cem Karadeniz², Timur \\ Meşe $^{2}$, Behzat Özkan ${ }^{1}$ \\ Divisions of ${ }^{1}$ Pediatric Endocrinology and ${ }^{2}$ Pediatric Cardiology, Department of Pediatrics, İzmir Dr. Behcet Uz Children's \\ Hospital, Izmir, Turkey. E-mail: drkorkmazanil@hotmail.com \\ Received: 11th April 2018, Revised: 9th June 2018, Accepted: 6th July 2018
}

SUMMARY: Korkmaz HA, Özdemir R, Küçük M, Karadeniz C, Meşe T, Özkan B. The impact of 21-hydroxylase deficiency on cardiac repolarization changes in children with 21-hydroxylase-deficient congenital adrenal hyperplasia. Turk J Pediatr 2019; 61: 228-235.

21-hydroxylase-deficient congenital adrenal hyperplasia (CAH) is associated with cardiovascular risk factors such as, hypertension, obesity, dyslipidemia, and insulin resistance. It is not known whether 21-hydroxylase-deficient CAH is risk factor for atrial and ventricular arrhythmias. The purpose of this study was to compare the 12-lead electrocardiographic measures in patients of 21-hydroxylase-deficient congenital adrenal hyperplasia with those in healthy control subjects matched for age, sex, height, weight and body mass index (BMI). Twenty-five patients with 21-hydroxylase-deficient $\mathrm{CAH}$ and twenty-five heathy control subjects were enrolled into this observational, cross-sectional, controlled study. The evaluation consisted of anthropometric measurements, biochemical parameters, and electrocardiographic (ECG) measures. The standard 12-lead electrocardiography was performed in all patients and P-wave dispersion (PWd), QT interval, QTd, QTcd, Tp-e dispersion, Tp-e/QT and Tp-e/QTc ratios were calculated. There were no significant differences in the groups for age, sex, height, weight and BMI (median age 9.4 (1.5-16.75) years, mean weight $37.6 \pm 21.5$ vs. $27.9 \pm 18.3 \mathrm{~kg}$, mean height $125.4 \pm 28.9$ vs. $114.7 \pm 31$ $\mathrm{cm}$, mean BMI $21.4 \pm 5.7$ vs. $18.9 \pm 3.4 \mathrm{~kg} / \mathrm{m}^{2}$, respectively). $\mathrm{P}$ dispersion and $\mathrm{Tp}$-e dispersion were significantly higher in patients of 21-hydroxylase-deficient CAH compared to the healthy subjects (median P dispersion 50 (25) vs. 40 (40) $\mathrm{ms}$, mean Tp-e dispersion $48 \pm 15.5$ vs. $35.2 \pm 17.5 \mathrm{~ms}$ ). Our study revealed that 21-hydroxylase deficient $\mathrm{CAH}$ is associated with high risk of atrial and ventricular arrhythmias in children.

Key words: ventricular arrhythmias, electrocardiographic measures, children, 21hydroxylase deficiency.

21-hydroxylase-deficient congenital adrenal hyperplasia $(\mathrm{CAH})$, an autosomal recessive condition, is associated with varying extent of impaired cortisol and aldosterone synthesis and adrenal androgen excess. ${ }^{1}$ Increase in ACTH secretion due to cortisol deficiency causes bilateral adrenal gland hyperplasia and raised earlier cortisol precursors below the mutant block. ${ }^{1}$ The dosage of glucocorticoids and mineralocorticoids for preventing adrenal crises should be carefully adjusted to avoid the effects of either over-treatment with glucocorticoids or overproduction adrenal androgens. ${ }^{2,3}$

A large number of electrocardiogram (ECG) derived indexes have been described for assessing the tendency for atrial and ventricular rhythm disturbances in various clinical conditions. Previously, the predictive value of P-wave dispersion (PWd), QT and corrected QT (QTc) intervals, QT and QTc dispersions for atrial, ventricular arrhythmias and sudden cardiac death (SCD) has been well described. -11 $^{-1}$ In recent years, T-wave peak-to-end (Tp-e) interval, which is the interval between the peak 
and the end of the T-wave on ECG, has emerged as a marker of the transmural dispersion of repolarization (TDR). Additionally, the Tp-e/QT and Tp-e/QTc ratios are used as an index for susceptibility to ventricular arrhythmogenesis.

Lifelong glucocorticoid treatment in patients with 21 hydroxylase deficiency may result in increased cardiovascular risk factors such as, obesity, insulin resistance with type 2 diabetes (T2DM), and hypertension. The early stage of hypertension was associated with an increased risk of myocardial fibrosis, ventricular hypertrophy and arrhythmias ${ }^{12}$. Glucocorticoids given in supraphysiological dose, play a role in myocardial fibrosis and cardiac hypertrophy with mineralocorticoid receptor (MR) activation. ${ }^{13-14}$ Myocardial fibrosis and remodeling may result in ventricular arrhythmias. To date, no study has been conducted to assess the impact of the disease and hydrocortisone treatment on electrocardiographic measures in children with 21-hydroxylase-deficient CAH. The purpose of this study is to assess the 12-lead electrocardiographic measures such as $\mathrm{PWd}$, QT interval, QTd, Tp-e interval, Tp-e/QT and Tp-e/QTc ratio in patients with 21-hydroxylasedeficient $\mathrm{CAH}$.

\section{Material and Methods}

\section{Patient population}

Twenty-five patients with 21-hydroxylasedeficient $\mathrm{CAH}$ and twenty-five age, sex and body size matched healthy control subjects were enrolled into this observational, crosssectional, controlled study. The children with 21-hydroxylase-deficient $\mathrm{CAH}$ were recruited consecutively from the outpatient clinic of the Pediatric Endocrinology, Dr. Behçet Uz Children's Hospital. 21-hydroxylase-deficient children who were recruited in this study were regularly followed at our institution from diagnosis, had adequate clinical and hormonal control, with a disease duration $>12$ months, and had no chronic diseases other than 21-hydroxylase-deficient CAH. Measurements included height and weight. The wall-mounted "Harpenden" stadiometer, which is similar to that designed by Tanner and Whitehouse, was used to measure height. Body mass index (BMI) was calculated $\left(\mathrm{kg} / \mathrm{m}^{2}\right)$. The standards of Greulich-Pyle method were used to assess bone age from radiographs of the left hand. At the time of diagnosis, all patients showed high basal levels of 17-hydroxyprogesterone (17-OHP) $(>50 \mu \mathrm{g} / \mathrm{L})$. The diagnosis of 21-hydroxylasedeficiency was confirmed by identification of homozygous or compound heterozygous CYP21A2 mutations. The patients were treated with hydrocortisone divided into three doses. The patients with salt wasting also received 9 - $\alpha$-fludrocortisone $0.1 \mathrm{mg} /$ day. None of the patients received additional medication.

The control group comprised children with no chronic illness. Written informed consent was acquired from the legal guardians of the children. The study was conducted according to the Declaration of Helsinki and the study protocol had been approved by Ethics Committee for Dr. Behcet Uz Children Hospital. The number of the document which verified approval of the study was 2014/06-02 (10.04.2014).

\section{Blood pressure measurements}

Blood pressure (BP) measurement was performed with using a conventional oscillatory measurement system positioned at the rightupper arm (DINAMAP; GE Healthcare, Munchen, Germany). The size of the cuff was selected for the patient's arm circumference, with the cuff bladder covering at least $40 \%$ and a maximum of $100 \%$ of the arm circumference. Standard deviation scores were obtained from normative values according to the literature. ${ }^{15}$

\section{Laboratory methods}

Blood samples were taken at 8:00 a.m. before the first dose of steroids and after an overnight fasting at least 12 hours. Fasting glucose, triglycerides, total cholesterol, and high-density lipoprotein (HDL) and low-density lipoprotein (LDL) cholesterol were measured by standard laboratory methods. Each sample was processed immediately after a maximum delay of 1 hour.

Adrenocorticotropic hormone (ACTH), plasma 17-OHP, serum cortisol, androstenedione and dehydroepiandrosterone sulphate (DHEA-S) were also measured. Serum insulin, androstenedione, ACTH and 17OHP measurements were performed with commercially available RIA kits (Siemens Healthcare Diagnostics, Inc., LA, CA, USA).

We calculated the homeostasis model assessment of insulin resistance (HOMA-IR) by using the formula: fasting insulin $(\mu \mathrm{U} / \mathrm{ml})$ 
$\mathrm{x}$ fasting glucose $(\mathrm{mmol} / \mathrm{L}) / 22.5{ }^{16}$

\section{Electrocardiography}

The standard 12-lead ECG (Cardiofax GEM, Model 9022 K; Nihon Kohden, Tokyo, Japan) was recorded at a speed of $25 \mathrm{~mm} / \mathrm{sec}$ and an amplitude of $1 \mathrm{mV} / \mathrm{cm}$ while in the supine position. ECG recordings were transferred to a personal computer. After 400x zooming in Adobe Photoshop software, measurements were performed directly from these ECG tracings by two pediatric cardiologists who were blinded to the patients files.

\section{$P$-wave dispersion}

P-wave duration was measured as the time between the beginning of the P-wave and the ending point of the P-wave. PWd was measured from the difference between the maximum and minimum P-wave durations. ${ }^{4}$

\section{QT dispersion}

The QT interval was measured from the onset of the QRS complex to the end of the T-wave. If $\mathrm{T}$-waves had two peaks and the second was smaller than $50 \%$ of the first one, then the point where the first peak reached the isoelectric line was considered the end of the T-wave. The corrected QT (QTc) interval was measured according to Bazzet's formula. ${ }^{17}$ The dispersion of the QT interval was obtained from the difference between the maximum and minimum QT interval.

\section{Tp-e interval}

The Tp-e interval was measured as duration from the peak of the T-wave and the end of the $\mathrm{T}$ - wave. If a lead contained inverted T-waves, we got the measurement from the lowest point of the inverted T-wave to its end. The U-wave was not taken into consideration. ${ }^{18}$ The dispersion of the Tp-e interval was measured as the differences between the maximum and the minimum Tp-e interval. The Tp-e/QT and Tp-e/QTc ratios were calculated from these measurements. ${ }^{19}$

\section{Follow-Up Protocol}

In every clinical visit, blood urea; serum creatinine; fasting glucose and insulin; serum albumin; bilirubin; aspartate aminotransferase; alanine aminotransferase; fasting serum lipid profile, including low-density lipoprotein; high-density lipoprotein; total cholesterol and triglycerides; serum 17-hydroxyprogesterone; DHEA-SO4; Androstenedione; and ACTH were measured. Patients with plasma levels of 17-hydroxyprogesterone $<10 \mu \mathrm{g} / \mathrm{L}$ was considered as having received adequate treatment and we defined them as the "controlled" group. Subjects with signs and symptoms of virilization and plasma levels of 17-hydroxyprogesterone above $10 \mu \mathrm{g} / \mathrm{L}$ were accepted as inadequately treated "uncontrolled" group. ${ }^{20}$

\section{Statistical analysis}

SPSS 22.0 (IBM Corporation, Armonk, NY, USA) was employed for data analysis. The Shapiro-Wilk test was performed to determine the conformity of the univariate data to normal distribution, and the Levene test was conducted to ascertain variance homogeneity. We carried out independent-samples t-test and Mann-Whitney U-tests to compare the two independent groups. Pearson and Spearman correlation tests were also employed to assess the strength of association between two variables. The quantitative data were expressed as median (minimum-maximum). The data were analysed at a 95\% confidence level and considered significant at a $\mathrm{P}$ value of $<0.05$.

\section{Results}

The median age of patients was 9.4 years (1.516.75), and the mean weight was 35.5 (7.5$76.3) \mathrm{kg}$. The median duration of treatment was 52.2 months (12-186 months). Patients' bone age, laboratory characteristics, mean hydrocortisone and fludrocortisone dosages are shown in Table I. Fifteen patients were receiving both hydroxycortisone and fludrocortisone, while 10 were on hydroxycortisone only. The median hydrocortisone dose was 18.8 (9.223.9) $\mathrm{mg} / \mathrm{m}^{2} /$ day. There was no significant correlation between hydrocortisone dose and systolic (P: 0.12) and diastolic (0.46) blood pressures. Also, the median systolic blood pressure and diastolic blood pressure in patients receiving 9-a-fludrocortisone were not different from those in patients on hydrocortisone only (P: 0.2). The CYP21A2 mutations included p.Q318X in seven patients, p.I172N in six, p.I2A in five, p.V28IL in three, p.R356W in two and 8-bp-deletion in two.

The patient group did not differ from the healthy group in terms of age, body mass index, diastolic blood pressure or plasma 
Table I. Laboratory Characteristics of the Patients.

\begin{tabular}{lc}
\hline Variables & median (minimum-maximum) \\
\hline Bone age, years & $12(1.5-17.5)$ \\
Chronologic age, years & $9.4(1.5-16.75)$ \\
Bone age/Chronologic age & $0.96(0.09-3.46)$ \\
DHEA-S, $\mu \mathrm{g} / \mathrm{ml}$ & $15(0.1-84)$ \\
Androstenedion, $\mathrm{ng} / \mathrm{ml}$ & $0.36(0.3-4.4)$ \\
Fasting insulin & $6.8(2.1-13.1)$ \\
Fasting glucose & $84(62-92)$ \\
HOMA-IR & $1.4(0.3-2.5)$ \\
Hydrocortisone dose, $\mathrm{mg} / \mathrm{m}^{2} / \mathrm{day}$ & $18.8(9.2-23.9)$ \\
Fludrocortisone dose, $\mathrm{mg} / \mathrm{day}$ & 0.1 \\
Duration of treatment, months & $53.2(12-186)$ \\
\hline
\end{tabular}

DHEA-S: dehydroepiandrosterone sulfate; HOMA-IR: homeostatic model assessment-insulin resistance;

levels of triglyceride, cholesterol, LDL and HDL. However, systolic blood pressure was significantly higher in the patient group compared to healthy subjects (Table II). Systolic blood pressure was positively correlated with weight $(\mathrm{p}<0.001, \mathrm{r}: 0.71)$, height (p: 0.001, r: 0.63), BMI $(p<0.001, r: 0.67)$, bone age $(\mathrm{p}<0.001, \mathrm{r}: 0.68)$, treatment duration ( $\mathrm{p}$ : 0.005 , r: 0.54), cholesterol (p: 0.01, r: 0.47), LDL cholesterol (p: 0.001, r: 0.61), insulin (p: 0.01, r: 0.5), and HOMA-IR (p: 0.01, r: 0.49) (Table III). Diastolic blood pressure was significantly positively correlated with weight $(p<0.001, r: 0.69)$, height $(p<0.001, r: 0.67)$,
BMI (p: 0.005, r: 0.54), bone age (p: 0.01, r: 0.46 ), treatment duration ( $\mathrm{p}: 0.005, \mathrm{r}: 0.54$ ) (Table III).

Table IV shows the electrocardiographic measures of the patients and the controls. The mean heart rate (beats/min), QT interval (ms), QT dispersion (ms), QTc (ms), QTc dispersion (ms), Tp-e interval (ms), Tp-e /QT and Tp-e/QTc were similar between patient and healthy groups. $\mathrm{P}$ dispersion and Tp-e dispersion were significantly higher in patients of 21-hydroxylase-deficient $\mathrm{CAH}$ compared to the healthy controls (median $\mathrm{P}$ dispersion 50 (25) vs. 40 (40) ms, mean Tp-e

Table II. Demographic, Clinical and Laboratory Characteristics of Patients and Controls

\begin{tabular}{lccc}
\hline Variables & Patients (n: 25) & Controls (n: 25) & $\mathrm{p}$ \\
\hline Age, years & $9.4(1.5-16.75)$ & $6.7(1.6-17.6)$ & 0.28 \\
Male/Female & $9 / 16$ & $8 / 17$ & \\
Height, cm & $131(75-163.5)$ & $116(79-174.6)$ & 0.21 \\
Height, SDS & $-0.1(-2.3-1.76)$ & $0(-1.1-1.67)$ & 0.67 \\
Weight, kg & $35.5(7.5-76.3)$ & $22(9-70)$ & 0.09 \\
Weight, SDS & $0.77(-2.7-4.3)$ & $0.4(-2-3)$ & 0.17 \\
Body mass index, kg/m² & $19.75(14.2-37.28)$ & $19(13-28.8)$ & 0.74 \\
Body mass index , SDS & $1.2(-1-2.72)$ & $0.8(-1.7-2.72)$ & 0.12 \\
Systolic blood pressure, mmHg & $100(80-130)$ & $90(78-120)$ & 0.008 \\
Diastolic blood pressure, mmHg & $65(40-80)$ & $60(55-85)$ & 0.41 \\
Triglyceride, mg/dl & $114(50-174)$ & $92(45-164)$ & 0.73 \\
Cholesterol, mg/dl & $160(114-195)$ & $160(100-211)$ & 0.47 \\
LDL, mg/dl & $90(64-116)$ & $87.8(65-112)$ & 0.47 \\
HDL, mg/dl & $46(36-83)$ & $50(35-82)$ & 0.32 \\
\hline
\end{tabular}

Variables are shown as median (minimum-maximum). HDL: high-density lipoprotein; LDL: low-density lipoprotein 

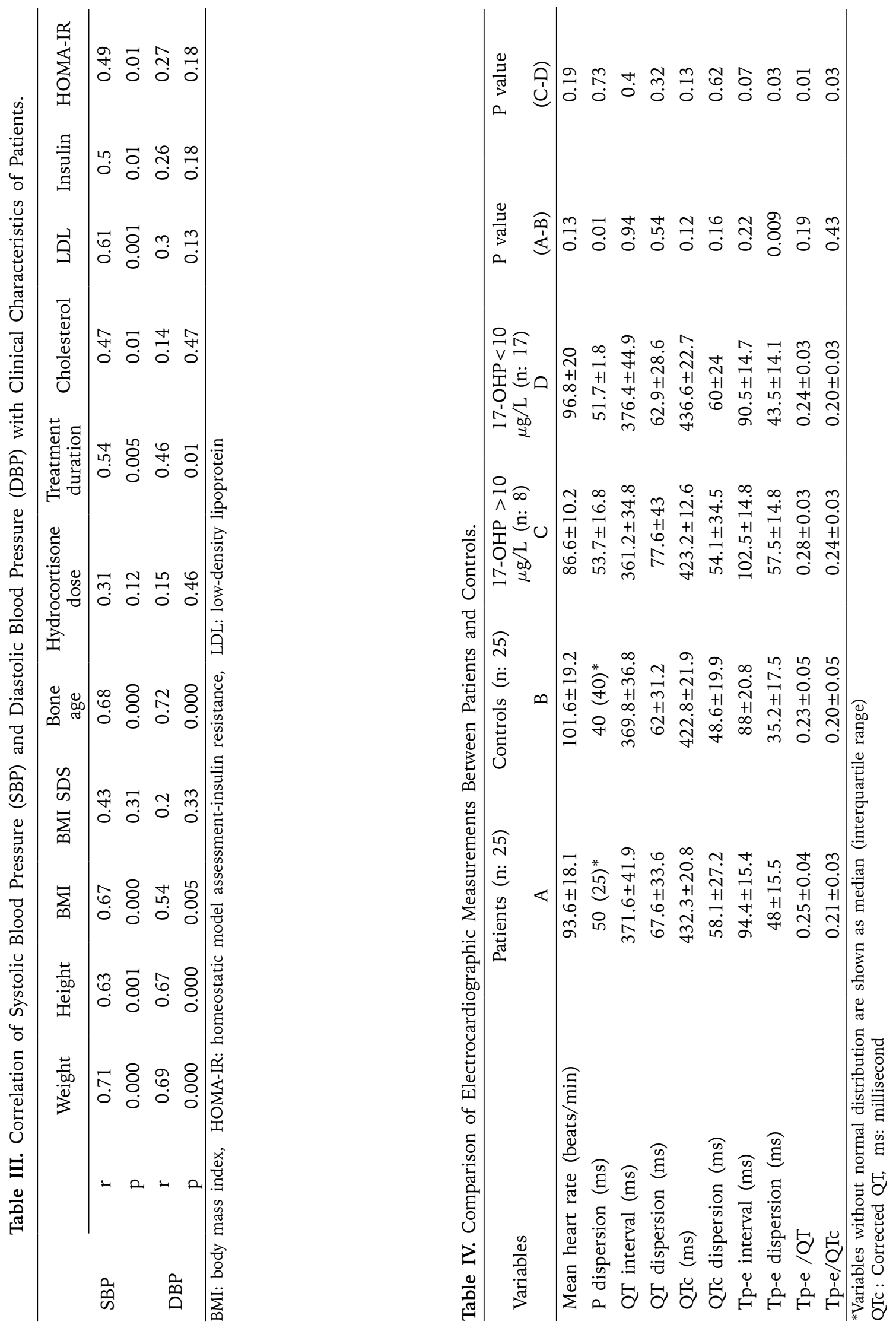
dispersion $48 \pm 15.5$ vs. $35.2 \pm 17.5 \mathrm{~ms}$ ) (Table IV). $\mathrm{P}$ dispersion was positively correlated with systolic blood pressure (p: 0.01, r: 0.489) and treatment duration ( $\mathrm{r}: 0.397, \mathrm{p}: 0.05$ ). T peakend dispersion was positively correlated with systolic blood pressure (p: 0.004, r: 0.559), BMI (p: 0.004, r: 0.551) and treatment duration (p: 0.02 , r: 0.455). According to the clinical and laboratory measures described above, eight patients were deemed to be uncontrolled. Tp-e dispersion (ms), Tp-e /QT and Tp-e/QTc were significantly higher in uncontrolled patients compared to the controlled patients (mean Tp-e dispersion $57.5 \pm 14.8$ vs. $43.5 \pm 14.1 \mathrm{~ms}$, mean Tp-e /QT $0.28 \pm 0.03$ vs. $0.24 \pm 0.03$, mean Tp-e/QTc $0.24 \pm 0.03$ vs. $0.20 \pm 0.03$ ) (Table IV).

\section{Discussion}

Researchers have found that 21-hydroxylase deficient $\mathrm{CAH}$ is a risk factor for hypertension, obesity, dyslipidemia, and insulin resistance.3,21-24 Our previous study found disturbance of ventricular functions in patients with $\mathrm{CAH}$, using tissue Doppler imaging techniques in these patients. ${ }^{25}$ However, data are still lacking regarding the effects of $\mathrm{CAH}$ on electrocardiographic measures in children with 21-hydroxylase-deficient CAH. In this study we found that 21-hydroxylase deficient $\mathrm{CAH}$ is associated with some adverse effects on electrocardiographic parameters among children and adolescents. To our knowledge, this study is the first to assess the increased risk for atrial and ventricular arrhythmias in children with 21-hydroxylase-deficient $\mathrm{CAH}$ using standard 12-lead electrocardiography.

In this study, we found a significant positive correlation between $\mathrm{T}$ peak-end dispersion and systolic blood pressure. We also found a significant positive correlation of $\mathrm{T}$ peak-end dispersion with hydroxycortisone treatment duration and BMI. These effects may be due to activated glucocorticoid and mineralocorticoid receptors on arterial and heart tissue, increased contractility of vascular tissue by enhancing sensitivity to noradrenaline and reduced endothelium dependent vasodilation. ${ }^{26}$ Ventricular cardiac arrhythmias have been considered as one of the first electrocardiographic abnormalities to appear in early hypertensive rats $^{12}$. Increased risk of high systolic blood pressure has been attributed to weight, BMI, duration of hydroxycortisone treatment, dyslipidemia, and insulin resistance. We found a significant correlation of high systolic blood pressure with hydroxycortisone treatment duration, dyslipidemia and insulin resistance in our study. Cortisol deficiency induces an increase in ACTH levels and results in high 17 hydroxy progesterone levels and adrenal androgen excess in uncontrolled patients with $\mathrm{CAH}$. High dose glucocorticoid replacement is required for suppressing high ACTH levels in uncontrolled patients, which contributes to high systolic blood pressure leading to ventricular hypertrophy inducing ventricular cardiac arrhythmias and death. A recent study demonstrated that children with $\mathrm{CAH}$ had left ventricular hypertrophy and impaired left ventricular diastolic dysfunction. ${ }^{20}$ We also found that patients with uncontrolled $\mathrm{CAH}$ had susceptibility for atrial and ventricular arrhythmias because of high dose glucocorticoid requirement, which suggested an association between $\mathrm{CAH}$ and risk of ventricular arrhythmias and death.

In this study, we found significant positive correlations between the duration of hydroxycortisone treatment and $\mathrm{P}$ dispersion, which indicated a predisposition to atrial arrhythmias. Previous studies regarding the relationship between corticosteroids and the risk of atrial fibrillation have been reported.27,28 Hydroxycortisone can have mineralocorticoid receptor (MR) associated with atrial fibrillation. ${ }^{27}$ Laszlo et al. ${ }^{29}$ demonstrated that MR antagonism stabilizes atrial electrophysiology by altering atrial ionic channels. Another mechanism of atrial fibrillation due to corticosteroids was a transient increase in serum potassium. ${ }^{30}$

Tp-e dispersion, which represents repolarization and modifications in normal pattern of ventricular recovery, has demonstrated to be effective to predict malignant arrhythmias in medical conditions. ${ }^{31,32}$ It has been proposed to be more useful than QTC and its dispersion in some clinical conditions. ${ }^{33} \mathrm{~A}$ recent study showed patients with uncontrolled $\mathrm{CAH}$ on medical treatment had more severe left ventricular hypertrophy and heart endothelial injury. ${ }^{20}$ We also found detrimental effects on ventricular functions in these uncontrolled patients. We reported increased susceptibility of 21-hydroxylase deficiency to ventricular 
cardiac arrhythmias and death in uncontrolled children and adolescents. Nguyen et al. ${ }^{12}$ found that interstitial myocardial fibrosis due to early hypertension was associated with adverse structural and electrical remodeling changes. Nguyen et al. ${ }^{12}$ hypothesized that basic differences in intracellular calcium cycling dynamics caused early after depolarization in ventricular myocytes from spontaneously hypertensive rats. Tp-e dispersion affected by levels of systolic blood pressure was significantly higher in the patient group and positively correlated with systolic blood pressure. $\mathrm{CAH}$ might exert its detrimental effects on ventricular arrhythmias by increasing blood pressure levels.

Our study had several limitations primarily being that it was an observational singleinstitution study that had a relatively small sample size. An additional limitation of our study might be a potential bias caused by inconsistent measurements of serum 17-hydroxyprogesterone, DHEA-SO4, Androstenedione and ACTH, because only data from patients in only one center with $\mathrm{CAH}$ were included in this study. Another limitation of our study is wide range of patients' age. Absence of 24-hour Holter monitoring and cardiac stress testing for the assessment of atrial and ventricular arrhythmias further limited the study. Performing 24-hour Holter monitoring and cardiac stress testing in patients with 21-hydroxylase deficient $\mathrm{CAH}$ would be interesting.

Nevertheless, this is the first study to show an association between atrial and ventricular arrhythmias and 21-hydroxylase deficient CAH. Our results demonstrated that children and adolescents with 21-hydroxylase-deficient $\mathrm{CAH}$ are more prone to some electrocardiographic changes which may lead to atrial and ventricular arrhythmias. Thus, these patients should be carefully evaluated with 12-lead ECG in terms of this electrocardiographic changes even in the absence of atrial and ventricular arrhythmias. Further prospective studies with large number of patients are needed to show prognostic value of these ECG parameters.

\section{REFERENCES}

1. Speiser PW, Azziz R, Baskin LS, et al; Endocrine Society. Congenital adrenal hyperplasia due to steroid 21- hydroxylase deficiency: an Endocrine Society clinical practice guideline. J Clin Endocrinol Metab 2010; 95: 4133-4160.

2. Arlt W, Willis DS, Wild SH, et al; United Kingdom Congenital Adrenal Hyperplasia Adult Study Executive (CaHASE). Health status of adults with congenital adrenal hyperplasia: a cohort study of 203 patients. J Clin Endocrinol Metab 2010; 95: 5110-5121.

3. Mooij CF, Kroese JM, Claahsen-van der Grinten HL, Tack CJ, Hermus ARMM. Unfavourable trends in cardiovascular and metabolic risk in paediatric and adult patients with congenital adrenal hyperplasia? Clin Endocrinol (Oxf) 2010; 73: 137-146.

4. Dilaveris PE, Gialafos JE. P-wave dispersion: a novel predictor of paroxysmal atrial fibrillation. Ann Noninvasive Electrocardiol 2001; 6: 159-165.

5. Seyfeli E, Duru M, Kuvandik G, Kaya H, Yalcin F. Effect of obesity on P-wave dispersion and QT dispersion in women. Int J Obes (Lond) 2006; 30: 957-961.

6. Amoozgar H, Ahmadipour M, Amirhakimi A. QT dispersion and $\mathrm{T}$ wave peak-to-end interval dispersion in children with Kawasaki disease. Int Cardiovasc Res J 2013; 7: 99-103.

7. Ulger Z, Aydinok Y, Levent E, Gurses D, Ozyurek AR. Evaluation of QT dispersion in beta thalassaemia major patients. Am J Hematol 2006; 81: 901-906.

8. Taşolar H, Ballı M, Bayramoğlu A, et al. Effect of smoking on Tp-e interval, Tp-e/QT and Tp-e/QTc ratios as indices of ventricular arrhythmogenesis. Heart Lung Circ 2014; 23: 827-832.

9. Bieganowska K, Sawicka-Parobczyk M, Bieganowski $\mathrm{M}$, Piskorski $\mathrm{J}$. $\mathrm{T}_{\text {peak }}-\mathrm{T}_{\text {end }}$ interval in 12-lead electrocardiogram of healthy children and adolescents $\mathrm{T}_{\text {peak }}-\mathrm{T}_{\text {end }}$ interval in childhood. Ann Noninvasive Electrocardiol 2013; 18: 344-351.

10. Sap F, Karataş Z, Altin H, et al. Dispersion durations of P-wave and QT interval in children with congenital heart disease and pulmonary arterial hypertension. Pediatr Cardiol 2013; 34: 591-596.

11. Yayla Ç, Bilgin M, Akboğa MK, et al. Evaluation of $\mathrm{Tp}-\mathrm{E}$ interval and Tp-E/QT ratio in patients with aortic stenosis. Ann Noninvasive Electrocardiol 2016; 21: 287-293.

12. Nguyen TP, Sovari AA, Pezhouman A, et al. Increased susceptibility of spontaneously hypertensive rats to ventricular tachyarrhythmias in early hypertension. J Physiol 2016; 594: 1689-1707.

13. Yasuoka S, Kai H, Kajimoto $\mathrm{H}$, et al. Blood pressure variability activates cardiac mineralocorticoid receptor and induces cardiac remodeling in hypertensive rats. Circ J 2013; 77: 1474-1481.

14. Johansen IB, Lunde IG, Røsjø H, et al. Cortisol response to stress is associated with myocardial remodeling in salmonid fishes. J Exp Biol 2011; 214(Pt 8): 13131321. 
15. de Man SA, Andre JL, Bachmann $\mathrm{H}$, et al. Blood pressure in childhood: pooled findings of six European studies. J Hypertens 1991; 9: 109-114.

16. Heinze E, Holl RW. Tests of cell function in childhood and adolescence. In: Ranke MB, (ed). Diagnostics of Endocrine Function in Children and Adolescents. Basel, Switzerland: Karger, 2003: 318-338.

17. Bazett HC. An analysis of the time-relations of electrocardiograms. Heart 1920; 7: 353-370.

18. Shimizu $\mathrm{M}$, Ino $\mathrm{H}$, Okeie $\mathrm{K}$, et al. T-peak to T-end interval may be a better predictor of high-risk patients with hypertrophic cardiomyopathy associated with a cardiac troponin I mutation than QT dispersion. Clin Cardiol 2002; 25: 335-339.

19. Kilicaslan F, Tokatli A, Ozdag F, et al. Tp-e interval, Tp-e/QT ratio, and Tp-e/QTc ratio are prolonged in patients with moderate and severe obstructive sleep apnea. Pacing Clin Electrophysiol 2012; 35: 966-972.

20. Metwalley KA, Farghaly HS, Sherief, T. Left ventricular dysfunction and subclinical atherosclerosis in children with classic congenital adrenal hyperplasia: a singlecenter study from upper Egypt. Eur J Pediatr 2016; 175: 405-412.

21. Mnif MF, Kamoun M, Mnif F, et al. Metabolic profile and cardiovascular risk factors in adult patients with congenital adrenal hyperplasia due to 21-hydroxylase deficiency. Indian J Endocrinol Metab 2012; 16: 939946.

22. Sartorato P, Zulian E, Benedini S, et al. Cardiovascular risk factors and ultrasound evaluation of intimamedia thickness at common carotids, carotid bulbs, and femoral and abdominal aorta arteries in patients with classic congenital adrenal hyperplasia due to 21-hydroxylase deficiency. J Clin Endocrinol Metab 2007; 92: 1015-1018.

23. Amr NH, Ahmed AY, Ibrahim YA. Carotid intima media thickness and other cardiovascular risk factors in children with congenital adrenal hyperplasia. J Endocrinol Invest 2014; 37: 1001-1008.
24. Zimmermann A, Grigorescu-Sido P, AlKhzouz C, et al. Alterations in lipid and carbohydrate metabolism in patients with classic congenital adrenal hyperplasia due to 21- hydroxylase deficiency. Horm Res Paediatr 2010; 74: 41-49.

25. Özdemir R, Korkmaz HA, Küçük M, Karadeniz C, Meşe T, Özkan B. Assessment of early atherosclerosis and left ventricular dysfunction in children with 21-hydroxylase deficiency. Clin Endocrinol (Oxf) 2017; 86: 473-479.

26. Walker BR. Glucocorticoids and cardiovascular disease. Eur J Endocrinol 2007; 157: 545-559.

27. van der Hooft CS, Heeringa J, Brusselle GG, et al. Corticosteroids and the risk of atrial fibrillation. Arch Intern Med 2006; 166: 1016-1020.

28. Hebb ALO, Imran SA, Morris DP, Bance M, Walling $S$. Presentation of atrial fibrillation following oral dexamethasone treatment in a NF2 patient. Am J Otolaryngol 2014; 35: 678-682.

29. Laszlo R, Bentz K, Schreieck J. Effects of aldosterone and mineralocorticoid receptor antagonism on cardiac ion channels in the view of upstream therapy of atrial fibrillation. Gen Physiol Biophys 2011; 30: 11-19.

30. McLuckie AE, Savage RW. Atrial fibrillation following pulse methylprednisolone therapy in an adult. Chest 1993; 104: 622-623.

31. Castro-Torres Y, Carmona-Puerta R, Katholi RE. Ventricular repolarization markers for predicting malignant arrhythmias in clinical practice. World J Clin Cases 2015; 3: 705-720.

32. Xia Y, Liang Y, Kongstad O, Holm M, Olsson B, Yuan S. Tpeak-Tend interval as an index of global dispersion of ventricular repolarization: evaluations using monophasic action potential mapping of the epiand endocardium in swine. J Interv Card Electrophysiol 2005; 14: 79-87.

33. Antzelevitch C, Shimizu W. Cellular mechanisms underlying the long QT syndrome. Curr Opin Cardiol 2002; 17: 43-51. 